Published in "NeuroImage 84: 615-625, 2014"

which should be cited to refer to this work.

\title{
A key region in the human parietal cortex for processing proprioceptive hand feedback during reaching movements
}

\author{
Alexandra Reichenbach a,b,*, Axel Thielscher ${ }^{\mathrm{a}, \mathrm{c}, \mathrm{d}}$, Angelika Peer ${ }^{\mathrm{e}}$, \\ Heinrich H. Bülthoff ${ }^{\text {a,f }}$, Jean-Pierre Bresciani ${ }^{\text {a,g,h }}$ \\ a Max Planck Institute for Biological Cybernetics, Tübingen, Germany \\ b Institute of Cognitive Neuroscience, University College London, UK \\ c Danish Research Center for Magnetic Resonance, Copenhagen University Hospital, Hvidovre, Denmark \\ d Biomedical Engineering Section, Technical University of Denmark, Kgs. Lyngby, Denmark \\ e Institute of Automatic Control Engineering, Technische Universität München, Germany \\ ${ }^{\mathrm{f}}$ Dept. of Brain and Cognitive Engineering, Korea University, Seoul, Republic of Korea \\ ${ }^{g}$ Laboratoire de Psychologie et Neurocognition CNRS UMR 5105, Université Pierre Mendès-France, Grenoble, France \\ h Department of Medicine, University of Fribourg, Fribourg, Switzerland
}

\begin{abstract}
Seemingly effortless, we adjust our movements to continuously changing environments. After initiation of a goal-directed movement, the motor command is under constant control of sensory feedback loops. The main sensory signals contributing to movement control are vision and proprioception. Recent neuroimaging studies have focused mainly on identifying the parts of the posterior parietal cortex (PPC) that contribute to visually guided movements. We used event-related TMS and force perturbations of the reaching hand to test whether the same sub-regions of the left PPC contribute to the processing of proprioceptive-only and of multi-sensory information about hand position when reaching for a visual target. TMS over two distinct stimulation sites elicited differential effects: TMS applied over the posterior part of the medial intraparietal sulcus (mIPS) compromised reaching accuracy when proprioception was the only sensory information available for correcting the reaching error. When visual feedback of the hand was available, TMS over the anterior intraparietal sulcus (aIPS) prolonged reaching time. Our results show for the first time the causal involvement of the posterior mIPS in processing proprioceptive feedback for online reaching control, and demonstrate that distinct cortical areas process proprioceptive-only and multi-sensory information for fast feedback corrections.
\end{abstract}

\section{Introduction}

During goal-directed movements, sensory information is continuously integrated into the motor plan in order to ensure and improve execution success (Desmurget and Grafton, 2000; Shadmehr et al., 2010; Wolpert et al., 1995). It is well established that the posterior parietal cortex (PPC) plays a prominent role in processing sensory information for motor control (Culham and Valyear, 2006; Filimon et al., 2009; Heed et al., 2011; Shadmehr and Krakauer, 2008), but the functional subdivisions within the human PPC are still highly debated.

Many neuroimaging studies on sensorimotor control have focused on processing visual information sources (Culham et al., 2006; Iacoboni, 2006), which provide information about the external world such as the reaching target, and about the moving hand. However, behavioral studies have shown that proprioception is nearly equally important for

* Corresponding author at: Institute of Cognitive Neuroscience, University College London, Alexandra House, 17 Queen Square, London WC1N 3AR, UK. Fax: +44 207916 8517.

E-mail address: a.reichenbach@ucl.ac.uk (A. Reichenbach). estimating the current hand position (Sarlegna et al., 2003, 2004). Furthermore, it has been suggested that the 'mode of control' of reaching movements to a visual target depends on whether only proprioceptive information about the hand position can be used, or whether vision is also available (Krakauer et al., 1999; Reichenbach et al., 2009). The latter allows for directly relating visual target and hand position in a common reference frame (Tagliabue and McIntyre, 2011), and different neural circuits may be recruited dependent on the mode of control (Bernier et al., 2009). Recent fMRI studies have assessed the relative contribution of different PPC areas to the processing of visual and proprioceptive information during motor control, mainly by investigating brain activity during the whole movement (Bernier and Grafton, 2010; Blangero et al., 2009; Filimon et al., 2009; Pellijeff et al., 2006), in which different temporal stages of movement control can hardly be disentangled. TMS studies have extended our current knowledge on this topic (Vesia and Crawford, 2012) to the temporal domain by focusing on the movement preparation phase (Vesia et al., 2008, 2010). As different mechanisms have been suggested for planning vs. execution of a goal-directed movement (Desmurget and Grafton, 2000; Glover, 2002; Sabes, 2000), their neural implementation may also rely on distinct cortical regions. 
For specifically studying the execution phase of a movement, or more specifically online control processes, random perturbations are introduced during the movement (Goodale et al., 1986), resulting in extremely fast changes of the movement. These perturbation paradigms are extensively used in behavioral research to investigate the integration of visual and proprioceptive afferent information into the current movement. On a cortical level, mainly the contribution of visual feedback to online control of reaching (Desmurget et al., 1999; Diedrichsen et al., 2005; Reichenbach et al., 2011) or grasping movements (Rice et al., 2006, 2007; Tunik et al., 2005, 2007) has been investigated. The temporal resolution of fMRI is sub-optimal for examining fast online control processes, and due to its correlative nature, one cannot assess whether the activated brain regions are crucial for those control processes. Thus, the cortical regions necessary for processing proprioceptive information for online reaching control remain elusive.

The goal of the current study was to delineate the parietal areas that are crucial for processing only proprioceptive feedback information during online control of goal-directed movements. Further, we aimed at comparing these regions with those contributing to multimodal processing of feedback information, i.e. vision and proprioception, again specifically for the online control phase. We utilized a reaching paradigm with force perturbations, which randomly pushed the reaching hand perpendicular to the reaching direction after the movement had started. Thus, participants could not anticipate the perturbation and had to correct online in order to reach the goal of the movement successfully. When visual feedback about the hand position was withdrawn, participants could only correct for the perturbation using proprioceptive information about their reaching hand position (proprioception-only). When visual feedback was available, they could use visual and proprioceptive information for correcting the reaching movement (proprioception + vision). Shortly after the force impulse, we applied event-related TMS on different parietal sites to probe the causal contribution of the underlying cortical areas to the corrective movements. The good temporal resolution of event-related TMS allowed us to interfere specifically with neural processing for the corrective movement. Behavioral deficits after administering TMS demonstrate a causal contribution of the stimulated cortical area to the correction process. In order to enhance the spatial resolution of TMS, we tested a whole grid of TMS stimulation sites (Busan et al., 2009, 2012; Reichenbach et al., 2011; Striemer et al., 2011) instead of only stimulating one likely candidate area and an unrelated control site.

The results of this study demonstrate for the first time the causal involvement of the posterior part of the medial intraparietal sulcus (mIPS) in processing of proprioception specifically for online control of reaching movements. The combined results of this and other studies (Chib et al., 2009; Della-Maggiore et al., 2004; Vesia et al., 2010) identify this region as a key area for processing proprioceptive information for motor control. Furthermore, this region can be clearly distinguished from an area around the anterior IPS, which is crucial for online sensorimotor processing in the presence of visual feedback about the hand position.

\section{Materials and methods}

\section{General procedure}

Nine healthy, right-handed volunteers (ages 21-32 years, four females) including one of the authors participated in the study. Besides the author, all participants were naive about the purpose of the study. They had normal or corrected-to-normal vision and no history of neurological disorders. Written informed consent was obtained from each participant prior to the first experiment. The study was conducted in accordance with the Declaration of Helsinki, and approved by the local ethics committee of the Medical Faculty of the University of Tübingen. Each participant attended several experimental sessions, in which he/ she was first familiarized with the overall procedure, then the pre- experiments were conducted, and finally the TMS experiment was performed in three sessions lasting $~ 2.5 \mathrm{~h}$ each, including setup. TMS sessions were separated by one week or more. During the MRI scan and the TMS experiments, participants wore earplugs to prevent hearing damage and auditory influence on task performance.

The experiments were conducted in a virtual reality environment with spatially matched visual and haptic scenes, where participants performed reaching movements to a visual target with their right hands holding onto a robot arm (Fig. 1A). During the movements, the robot arm applied brief force perturbations to the reaching hand (Fig. 1B) under two sensory feedback conditions. Proprioception-only: Visual feedback about the hand position was not available, thus participants could only use proprioceptive information about the hand position for guiding the movement, and for correcting trajectory deviations induced by the force perturbation. Proprioception + vision: Participants could use both visual and proprioceptive feedback about the hand position for correcting the arm trajectory. We then tested whether the participants' ability to correct for the perturbation was reduced when event-related TMS was applied to nine distinct brain regions over the anterior part of the posterior parietal cortex (PPC). We chose the stimulation sites such that they covered approximately equidistantly the left PPC, including areas that are involved in online movement control or the processing of proprioceptive information according to previous studies (Table 1 ).

\section{Pre-experiments}

A T1-weighted structural image (MPRAGE, TR $1900 \mathrm{~ms}$, TE $2.26 \mathrm{~ms}$, TI $900 \mathrm{~ms}$, flip angle $9^{\circ}, 192$ coronal slices, $1 \mathrm{~mm}$ iso-voxel resolution, 2 averages) was acquired on a Siemens 3T TIM Trio (Siemens, Erlangen, Germany) for each participant. These images were later used to position the TMS coil.

In a behavioral session prior to the TMS sessions, we determined the individual force levels needed to equate the impact of the force perturbation across participants. The goal was to match the resulting reaching trajectories between participants by matching the maximal trajectory deviation resulting from the force perturbation. Participants performed the same task as in the main experiment (see below) without TMS stimulation, but with force impulses of different strengths intermixed, ranging from 0 to $25 \mathrm{~N}$ in steps of $5 \mathrm{~N}$. The force level resulting in a maximal trajectory deviation closest to $25 \mathrm{~mm}$ was used as individual force level. This procedure yielded force levels of $10 \mathrm{~N}$ for two participants and $15 \mathrm{~N}$ for the remaining seven. Additionally, this session served to minimize training effects in the TMS sessions.

\section{Technical Setup}

A mirror-setup with a top-mounted CRT monitor and shutter glasses (StereoGraphics/REAL D, Beverly Hills, California, USA) was used to render the visual scene in 3D in congruence to the haptic scene (Fig. 1, Supplementary material Figure S1). The latter was controlled by a robot arm (DekiFeD, Technische Universität München, Germany; (Buss and Schmidt, 1999)) used as manipulandum, which restricted the hand movements to a horizontal plane with two degrees of freedom. Participants were comfortably seated on a chair, and a chin and forehead rest supported their head in order to minimize head movements. They kept a handle, which was mounted on top of the robot arm, grasped with their right hand throughout the experimental blocks. Visual feedback about the hand position (a red sphere) projected onto the mirror corresponded spatially to the top of this handle, but the mirror prevented direct vision on the hand itself. Thus, the visual scene provided veridical feedback about the physical hand position not requiring additional coordinate transformations between visual and proprioceptive hand feedback. The robot arm actively followed the hand movements to minimize its inertia as felt by the participant. Visual scene presentation 


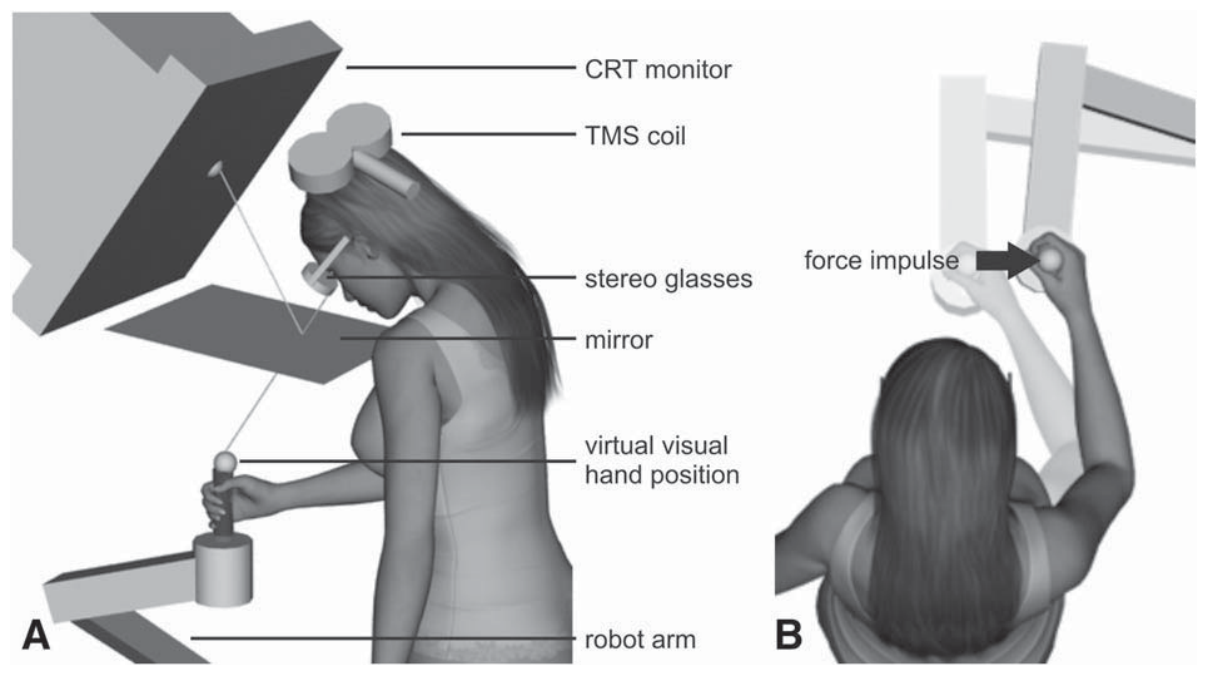

Fig. 1. Schematics of the experimental setup. A) Illustration of the visual and the matching haptic scene, with the latter being occluded by the mirror. The TMS coil position roughly corresponds to a stimulation site above the left PPC. Not shown: The participant was seated comfortably in a chair with his head supported by a chin and a forehead rest to minimized head movements (cf. Supplementary material Figure S1). B) Illustration of the force perturbation.

and acquisition of the kinematic data was performed at $120 \mathrm{~Hz}$. For additional technical details, please refer to Reichenbach et al. (2009).

\section{Procedure and behavioral task}

Each of the three TMS sessions proceeded in complete darkness, except for the visual objects rendered on the CRT monitor on a black background. Each session consisted of 12 experimental blocks, plus one initial training block to familiarize the participant with the task and to minimize training effects throughout the experiment. An experimental block lasted 10-15 min and contained 72 trials that covered three repetitions of all possible combinations of the independent variables (except for TMS stimulation site being constant for each block) to equal proportions: Sensory feedback about the hand position (proprioception-only/proprioception + vision), TMS (yes/no), target position ( $15^{\circ}$ to the left $/ 15^{\circ}$ to the right), and force perturbation (to the right/ to the left/none). The order of presentation was fully randomized in a block to prevent any predictability or anticipation of the force

\section{Table 1}

TMS stimulation sites. The MNI coordinates used to plan the TMS stimulation sites are specified, additionally to the corresponding references. The coordinates from the study of Reichenbach et al. (2011) are all brain areas where the authors found significant reach-related BOLD activity, or activity related to online corrections to visual perturbations. Additionally, TMS over the sites aIPS, $S M G_{\text {superior, }}$ and $S M G_{\text {middle }}$ yielded significant disturbance of the correction to visual perturbations in that study. mIPS $_{\text {posterior }}$ was included because two TMS studies successfully used this stimulation site to interfere with the processing of proprioceptive hand/arm information during a motor task (Chib et al.,

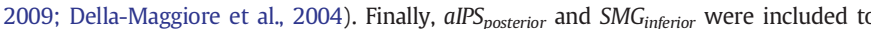
complement the grid. The tested sites constitute a compromise between feasibility of the study with respect to the testing time and covering the portions of the PPC that might be involved in online processing of sensory information about the reaching hand/ arm. We did not include more medial-posterior areas as they have mainly been associated with processing information about the target of reach (Bernier and Grafton, 2010 Diedrichsen et al., 2005; Gallivan et al., 2009; Vesia et al., 2010).

\begin{tabular}{|c|c|c|}
\hline $\begin{array}{l}\text { Stimulation } \\
\text { Site }\end{array}$ & $\begin{array}{l}\text { MNI coordinates } \\
\mathrm{x} / \mathrm{y} / \mathrm{z} \text { in }[\mathrm{mm}]\end{array}$ & Derived from \\
\hline mIPS $_{\text {anterior }}$ & $-36 /-49 / 57$ & Stimulation site $S P L_{\text {group }}$ (Reichenbach et al., 2011) \\
\hline mIPS $_{\text {middle }}$ & $-33 /-56 / 55$ & Stimulation site reach (Reichenbach et al., 2011) \\
\hline mIPS $_{\text {posterior }}$ & $-36 /-64 / 54$ & (Chib et al., 2009; Della-Maggiore et al., 2004) \\
\hline aIPS & $-44 /-42 / 55$ & Stimulation site IPS group (Reichenbach et al., 2011) \\
\hline aIPS $_{\text {posterior }}$ & $-41 /-49 / 53$ & Same vector from aIPS as mIPS $_{\text {middle }}$ from mIPS anterior \\
\hline $\mathrm{SMG}_{\text {superior }}$ & $-45 /-40 / 45$ & Stimulation site $S M G_{\text {group }}$ (Reichenbach et al., 2011) \\
\hline $\mathrm{SMG}_{\text {middle }}$ & $-53 /-33 / 40$ & Stimulation site $S M G_{\text {indiv }}$ (Reichenbach et al., 2011) \\
\hline $\mathrm{SMG}_{\text {inferior }}$ & $-60 /-27 / 36$ & Anterior-inferior to $\mathrm{SMG}_{\text {middle }}$ \\
\hline aIPS $_{\text {right }}$ & $44 /-42 / 57$ & Stimulation site $I P S_{\text {right }}$ (Reichenbach et al., 2011) \\
\hline
\end{tabular}

perturbation and the administration of TMS. Altogether, 12 repetitions were recorded for each combination of independent variables (including the TMS stimulation site), resulting in four experimental blocks per TMS stimulation site. The order of the 36 blocks was pseudorandomized within and across sessions for each participant independently to prevent putative training or fatigue effects from biasing the results. Pseudo-randomization in the respect that each set of stimulation sites was tested in a fully randomized fashion before proceeding with the next set of stimulation sites with the additional constraint that no site was tested twice in a row. Post-experiment interviews confirmed that the stimulation sites over the left PPC were undistinguishable for the participants.

A trial started with the presentation of the starting position (magenta sphere) with visual feedback about the hand position (red sphere) present. The starting position was randomly jittered in a $2 \times 2 \mathrm{~cm}$ area located $10 \mathrm{~cm}$ in front of the participant about the body midline. After the hand had been maintained in the starting position for about one second, the target (another magenta sphere) appeared and the starting position disappeared. The target was displayed at a distance of $20 \mathrm{~cm}$ from the starting position and its location was $15^{\circ}$ on either side of the body midline. The participant's task was to look at and reach for the target as quickly and precisely as possible. In proprioception-only reaching trials, the visual feedback about the hand position disappeared as soon as the target appeared. The time at which the velocity of the hand dropped below $1 \mathrm{~cm} / \mathrm{s}$ again was defined as the end of the trial, after which the visual scene disappeared for two seconds.

In force perturbation trials, the robot arm exerted a constant force on the participants' hand over a reaching distance of $5 \mathrm{~cm}$. The onset of the force was randomized between 1 and $4 \mathrm{~cm}$ away from the starting position, and the direction was perpendicular to the reaching direction to either side. In TMS trials, four magnetic pulses were applied at a frequency of $60 \mathrm{~Hz}$. The first pulse was delivered $30 \mathrm{~ms}$ after onset of the force impulse (Reichenbach et al., 2009, 2011). Since the first corrective responses to such perturbations can be observed with EMG within less than 100 ms (Reichenbach et al., 2009), we covered with the TMS pulses (ranging from 30 to $90 \mathrm{~ms}$ after the perturbation started) the whole period in which the processing for initiating the correction occurred.

\section{TMS stimulation}

A grid of 8 TMS coil positions ranging from the inferior SMG to the posterior part of the MIPS in the left hemisphere (Fig. 2), and one additional position on the contra-lateral hemisphere were derived from 


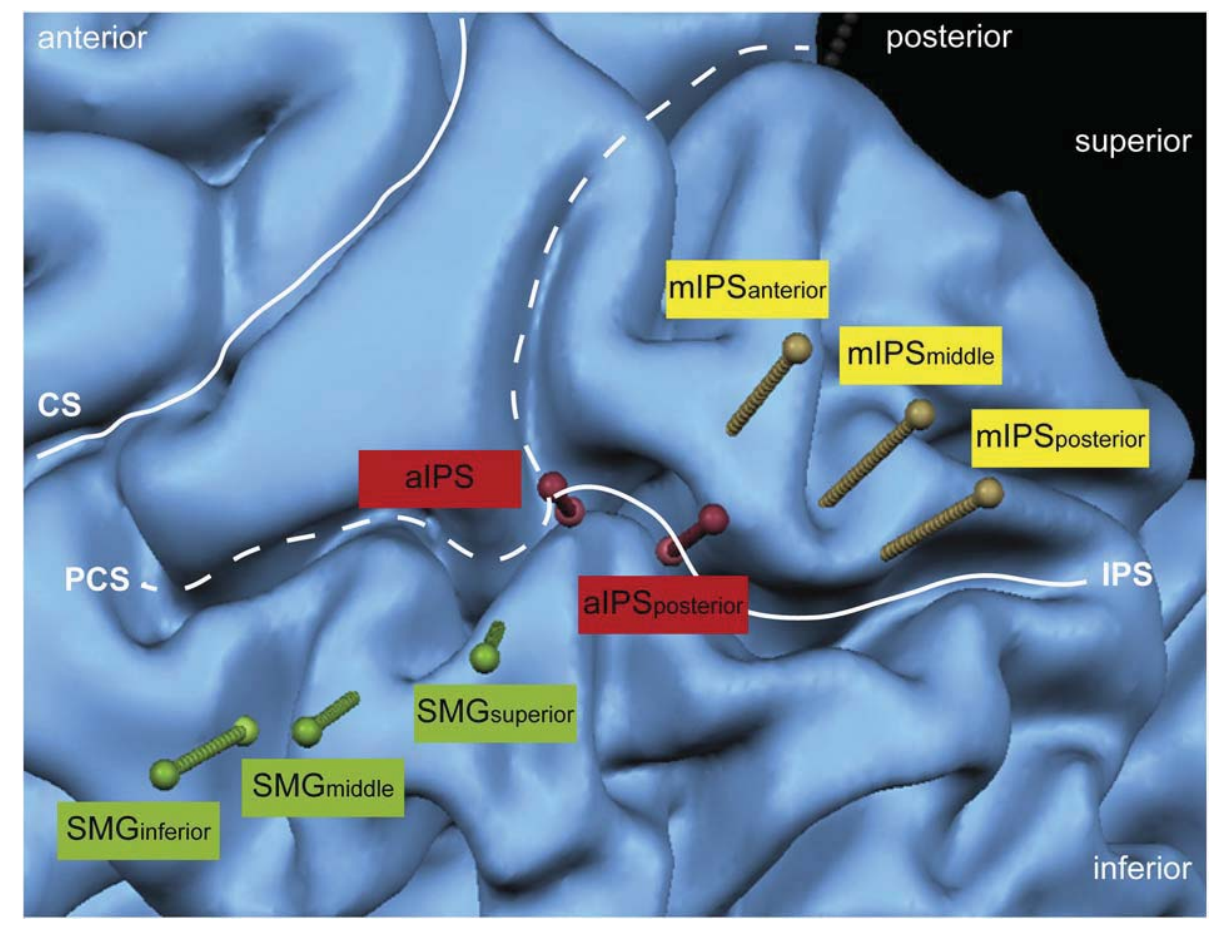

Fig. 2. TMS stimulation sites, rendered on the reconstructed left hemisphere of an exemplarily participant. Adjacent stimulation sites were $0.5-1.5$ cm apart. CS: central sulcus; PCS: postcentral sulcus; IPS: intraparietal sulcus; mIPS: medial IPS; aIPS: anterior IPS; SMG: supramarginal gyrus.

(a)

previous TMS studies investigating online control or proprioceptive processing during reaching (Chib et al., 2009; Della-Maggiore et al., 2004; Reichenbach et al., 2011) (Table 1). With these sites, we covered broad region of the left PPC for which sensorimotor processing related to our questions had been localized previously. The extra control site on the right hemisphere was added to match unspecific side effects and the discomfort of the most anterior stimulation site (aIPS), to which this control site was mirrored. The distance between adjacent stimulation sites ranged between 0.5 and $1.5 \mathrm{~cm}$, dependent on the sites and the participants' head size. This allowed dissociation between different sub-regions of the PPC, and adjacent coil positions served as mutual control sites. Individual coil positions were preplanned by transforming the MNI coordinates of the grid from MNI space (Mazziotta et al., 2001) to the space of the individual structural images using the linear registration (FLIRT) of FSL 4.0 (FMRIB, Oxford, UK; (Smith et al., 2004, 2005)). The closest coil position on the skull of every participant was determined for each coil position using custom-written MATLAB routines (The MathWorks, Natick, MA, USA) and the surface reconstruction of the skull as obtained with BrainVoyager 2000 (Brain Innovation, Maastricht, The Netherlands).

Biphasic TMS stimuli were applied with standard current direction using a Medtronic MagPro X100 stimulator (MagVenture, Farum, Denmark) with a MC-B70 butterfly coil. The coil position was monitored using a neuronavigation system (BrainView, Fraunhofer IPA, Germany; see Kammer et al. (2007) for a description of the system). The spatial accuracy of the registration between the participant's real head and his anatomical MR image in the neuronavigation system was established at the beginning, and checked again at the end of each session using positions of clearly visible landmarks (e.g., nasion and inion). A trained investigator held the coil manually, keeping the coil position in a range of $2 \mathrm{~mm}$ to the pre-planned stimulation sites on the skull by constantly monitoring the coil position on the display of the neuronavigation system that also displayed the numerical distance between the planned and the actual coil position in mm. Blocks were repeated (five blocks total) whenever the distance of the coil to the planned stimulation site exceeded $2 \mathrm{~mm}$. The stimulation intensity was chosen to meet two competing goals: It should be as high as possible to maximize the impact on the stimulation site without eliciting direct effects on the primary motor cortex. For this purpose, the coil was placed at the most anterior stimulation site at the beginning of each session, and the intensity was gradually decreased until no more motor responses were elicited in the finger muscles for at least 10 consecutive trials (tested by recording surface EMG from the relaxed first dorsal interosseus). To ensure no stimulation of the primary motor cortex during the movements, $80 \%$ of this intensity was then used as the individual stimulation intensity throughout the session. This procedure resulted in stimulation intensities of 32-61\% of maximum stimulator output. The coil was initially oriented parallel to the central sulcus with the handle pointing downwards, and adjusted when necessary (see Supplementary material Table S1 for details). The induced field direction of the most effective flank was therefore directed from posterior-lateral to anterior-medial, perpendicular to the central sulcus.

\section{Behavioral measures and data analysis}

The on- and offset of the movement for data analysis purposes were defined as the time at which the velocity of the robot arm exceeded and fell below $2 \mathrm{~cm} / \mathrm{s}$, respectively. Trials were excluded as outliers from further analysis if total time, total path length, or peak velocity were outside the range of the participant's mean $\pm 3 * \mathrm{SD}$. Thus, the subsequent data analyses included $98 \%$ of the recorded trials.

The impact of TMS on the online corrections to the force perturbation was assessed by applying different measures to the kinematic data. Endpoint error was defined as the distance of the final hand position to the target and calculated separately for the component perpendicular to the reaching direction towards the targets, and for the component in direction of the reaching targets. For the force perturbed trials, the error perpendicular to the reaching direction denoted the amount of correction for the perturbation, and was thus coded with respect to the perturbation (CorrX). Incomplete corrections for the force impulse were coded with positive values, and overshooting corrections would then be denoted with negative values. For unperturbed trials, the error perpendicular to the reaching direction was the actual deviations 
from the target (ErrorX), with rightwards deviations coded with positive values. For the errors in direction of the reaching, overshooting the target was coded as positive value (ErrorY), both for perturbed and unperturbed movements. As the perturbation occurred perpendicular to the reach, we did not expect a correction in the direction of the reach. For both directional errors, we also assessed the variability (VarX, VarY), which was defined as the within-subject standard deviation of the endpoint measures. As an additional spatial measure, we assessed for the perturbed trials the absolute value of the maximum deviation (MaxDev) between the recorded trajectory and an 'ideal trajectory' (i.e., a straight line between the starting point and the target). This measure was mainly used to control for successful manipulation of the trajectory by the force impulse. The overall reaching time (ReachTime) was split up in acceleration (AccTime) and deceleration (DecTime) time, separated by the time point when the maximal velocity was reached. We conducted the main analyses for the trials with force perturbations to assess the effect of TMS on the online corrections. To control for general reaching related effects of TMS, most analyses were also conducted for the trials without force perturbation. The interest of this study focused on general effects of TMS on processing sensory hand information for the online control of reaching movements independent of the hemifield of the target or the direction of the correction. Thus, we first confirmed with a detailed analysis on our main measure of interest, CorrX, that TMS did not differentially influence the corrective responses dependent on target or perturbation direction. Subsequently, we collapsed the data across the factors of no interest target position ( $15^{\circ}$ to the left or to the right) and perturbation direction (to the left or right) for all further analyses.

For each TMS stimulation site, we first compared trials with versus without TMS using pre-planned paired t-tests separately for trials with and without availability of the visual feedback about the hand position. To correct for multiple comparisons, we used Bonferroni corrections for the number of stimulation sites. Additionally, we conducted permutation tests with 10000 repetitions in order to assess the $p$-values that would yield at most $5 \%$ false positive accumulated over all stimulation sites given our data (see Supplementary material S1 for a detailed description of the method). In order to confirm any significant effects from this first- level analysis, we compared the TMS effects (TMS-no TMS) of these sites to the average TMS effect of all other sites (Oliver et al., 2009). As next step, to test for the spatial specificity of a stimulation site that showed a significant effect, we compared the TMS effect for this site with its adjacent stimulation sites (all sites closer than $1.5 \mathrm{~cm}$ ). Finally, to test for the functional specificity of this site, we compared the TMS effects between the two sensory feedback conditions for this site. Compared to a standard omnibus ANOVA, this approach has a higher power to detect significant effects that are localized to a single stimulation site when testing a larger number of sites. Reported values are mean \pm SE across participants, unless stated otherwise. Bonferroni-corrected t-tests and the corresponding permutation tests converged to the same results. Therefore, we only report the values for the permutation tests.

\section{Control measurements}

The individual force strengths led to a MaxDev around $25 \mathrm{~mm}$ and a small inter-individual variance (Table 2). Thus, the experimental manipulation to equate the spatial deviation by using individual force strengths was accomplished successfully. For none of the stimulation sites did TMS affect MaxDev in any condition.

The force impulse started on average $99.5 \mathrm{~ms}$ after movement onset with an intra-subject standard deviation of $25.5 \mathrm{~ms}$. Post-hoc comparisons revealed that neither intra- nor inter-subjects differences in the onset of the force impulse were present for the conditions with TMS effects. Thus, none of the effects reported can be attributed to differences in onset of the force impulse between conditions.

Re-analysis of the data without the participating author did not change any of the effects as reported in the Results section.

\section{Results}

\section{Reaching behavior averaged across TMS stimulation sites}

All participants showed fast responses to the force perturbations and corrected appropriately (Fig. 3, Supplementary material Figure S2). The force perturbation prolonged the overall reaching time from 599 to

\section{Table 2}

Average values and general effects across stimulation sites for all conditions. Positive values of CorrX represent incomplete compensation for the force perturbation, negative values would denote an over-compensation. For the unperturbed movements, positive ErrorX values denote a deviation to the right. Positive values of ErrorY denote an overshoot with respect to the target. The mean $( \pm$ SE) across participants is given. The comparisons display the main effects for sensory feedback (proprioception-only $v s$. proprioception + vision) and TMS (TMS vs. no TMS) of the measures directly above, separately for force-perturbed and unperturbed reaching. The main effect for the force perturbation compares the force-perturbed $v s$. unperturbed trials. Statistical significant comparisons $(p<.05)$ are emphasized bold. Note that CorrX and ErrorX have different meanings and can thus not be compared directly.

\begin{tabular}{|c|c|c|c|c|c|c|c|}
\hline \multicolumn{8}{|l|}{ Force-perturbed } \\
\hline & & CorrX [mm] & $\operatorname{VarX}[\mathrm{mm}]$ & ErrorY [mm] & $\operatorname{VarY}[\mathrm{mm}]$ & Reach Time [ms] & Max Dev [mm] \\
\hline \multirow[t]{2}{*}{ Proprioception-only } & TMS & $3.0(1.3)$ & $12.3(1.1)$ & $29.6(6.0)$ & $11.7(1.0)$ & $667.5(32.4)$ & $23.9(1.1)$ \\
\hline & no TMS & $2.2(1.8)$ & $12.3(1.0)$ & $28.7(5.4)$ & $11.7(1.0)$ & $666.1(32.1)$ & $23.5(1.2)$ \\
\hline \multirow[t]{2}{*}{ Proprioception + vision } & TMS & $0.3(0.8)$ & $6.8(0.7)$ & $0.7(0.8)$ & $6.7(0.6)$ & $671.2(23.2)$ & $23.3(1.4)$ \\
\hline & no TMS & $0.5(0.8)$ & $6.7(0.7)$ & $0.6(0.9)$ & $6.5(0.7)$ & $667.2(24.5)$ & $23.1(1.4)$ \\
\hline \multirow[t]{2}{*}{ Sensory feedback effect } & & $t_{8}=1.98$ & $t_{8}=5.22$ & $t_{8}=5.44$ & $t_{8}=7.38$ & $t_{8}=0.22$ & \\
\hline & & $p>.05$ & $p<.001$ & $p<.001$ & $p<.001$ & $\mathrm{p}>.8$ & \\
\hline \multirow[t]{2}{*}{ TMS effect } & & $t_{8}=1.92$ & $t_{8}=0.35$ & $t_{8}=0.90$ & $t_{8}=0.41$ & $t_{8}=1.40$ & \\
\hline & & $p>.05$ & $p>.7$ & $p>.3$ & $p>.6$ & $p>.2$ & \\
\hline
\end{tabular}

Unperturbed

\begin{tabular}{|c|c|c|c|c|c|c|}
\hline & & ErrorX [mm] & $\operatorname{VarX}[\mathrm{mm}]$ & ErrorY [mm] & $\operatorname{VarY}[\mathrm{mm}]$ & Reach Time [ms] \\
\hline \multirow[t]{2}{*}{ Proprioception-only } & TMS & $6.0(2.8)$ & $10.8(1.0)$ & $30.6(5.7)$ & $12.2(1.1)$ & $605.9(27.6)$ \\
\hline & no TMS & $6.6(3.0)$ & $10.7(1.1)$ & $30.2(5.4)$ & $12.2(1.2)$ & $600.6(23.9)$ \\
\hline \multirow[t]{2}{*}{ Proprioception + vision } & TMS & $-0.2(0.3)$ & $5.8(0.5)$ & $3.7(1.5)$ & $8.3(1.0)$ & $595.6(18.4)$ \\
\hline & no TMS & $-0.4(0.4)$ & $5.8(0.6)$ & $3.3(1.4)$ & $8.2(1.0)$ & $594.1(18.9)$ \\
\hline \multirow{2}{*}{\multicolumn{2}{|c|}{ Sensory feedback effect }} & $t_{8}=2.12$ & $t_{8}=6.28$ & $t_{8}=5.65$ & $t_{8}=6.29$ & $t_{8}=0.56$ \\
\hline & & $p>.05$ & $\mathbf{p}<.001$ & $\mathrm{p}<.001$ & $\mathrm{p}<.001$ & $p>.5$ \\
\hline \multirow{2}{*}{\multicolumn{2}{|c|}{ TMS effect }} & $t_{8}=0.88$ & $t_{8}=0.01$ & $t_{8}=0.81$ & $t_{8}=0.12$ & $t_{8}=1.18$ \\
\hline & & $p>.4$ & $p>.9$ & $p>.4$ & $p>.9$ & $p>.2$ \\
\hline \multicolumn{2}{|l|}{ Force perturbation effect } & Not matched & $\begin{array}{l}t_{8}=3.74 \\
p<.01\end{array}$ & $\begin{array}{l}t_{8}=1.75 \\
p>.1\end{array}$ & $\begin{array}{l}t_{8}=3.31 \\
p<.01\end{array}$ & $\begin{array}{l}t_{8}=4.92 \\
p<.01\end{array}$ \\
\hline
\end{tabular}


A mIPS posterior: path normalized; proprioception-only

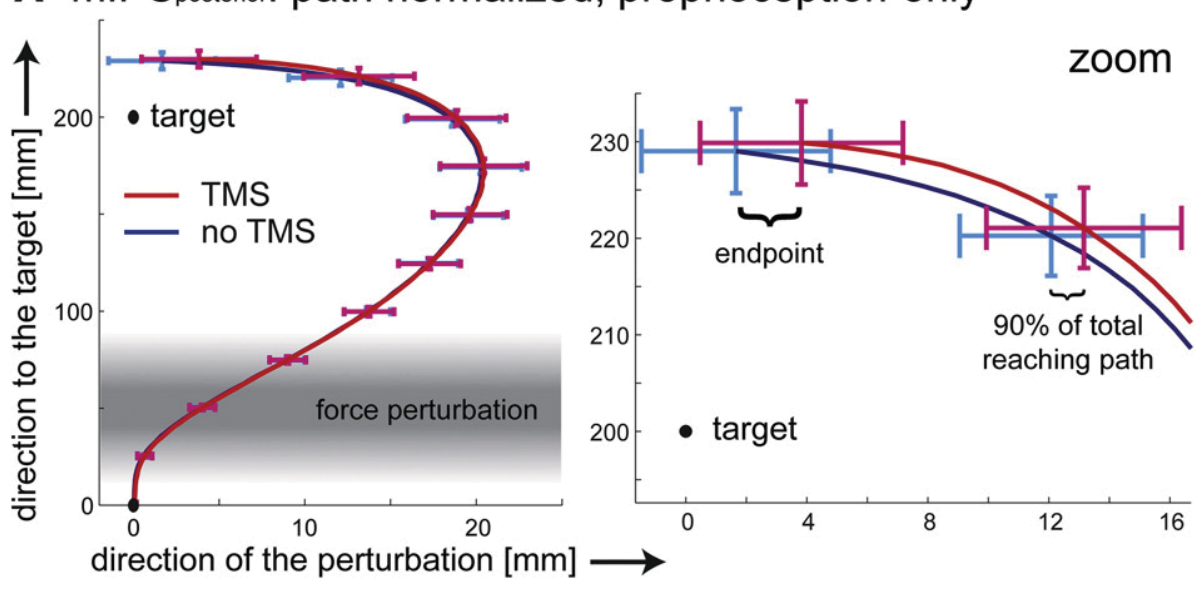

B alPS: time normalized; proprioception+vision
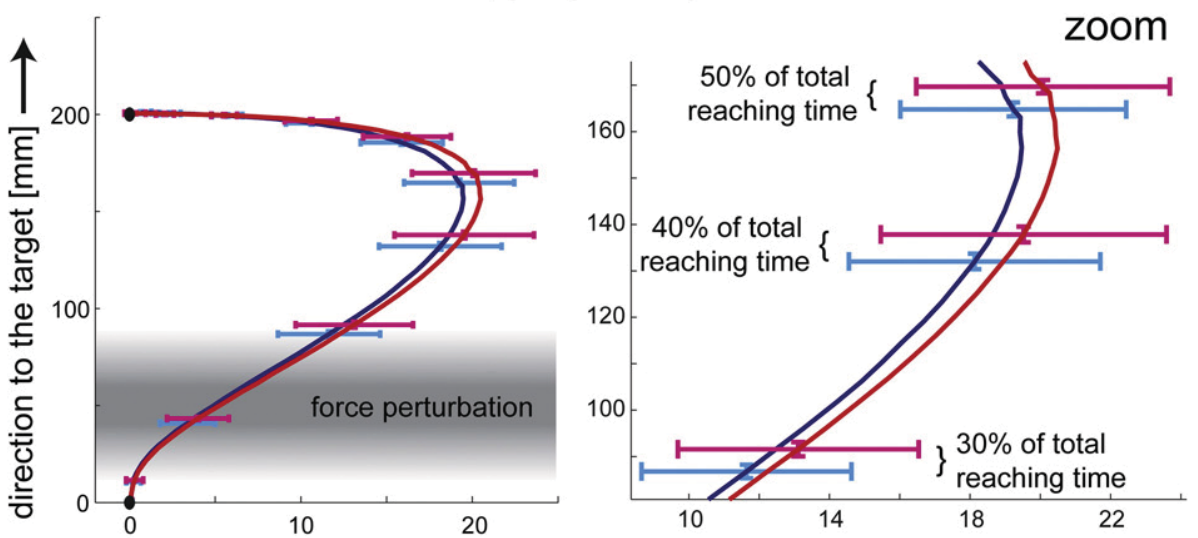

direction of the perturbation $[\mathrm{mm}] \longrightarrow$
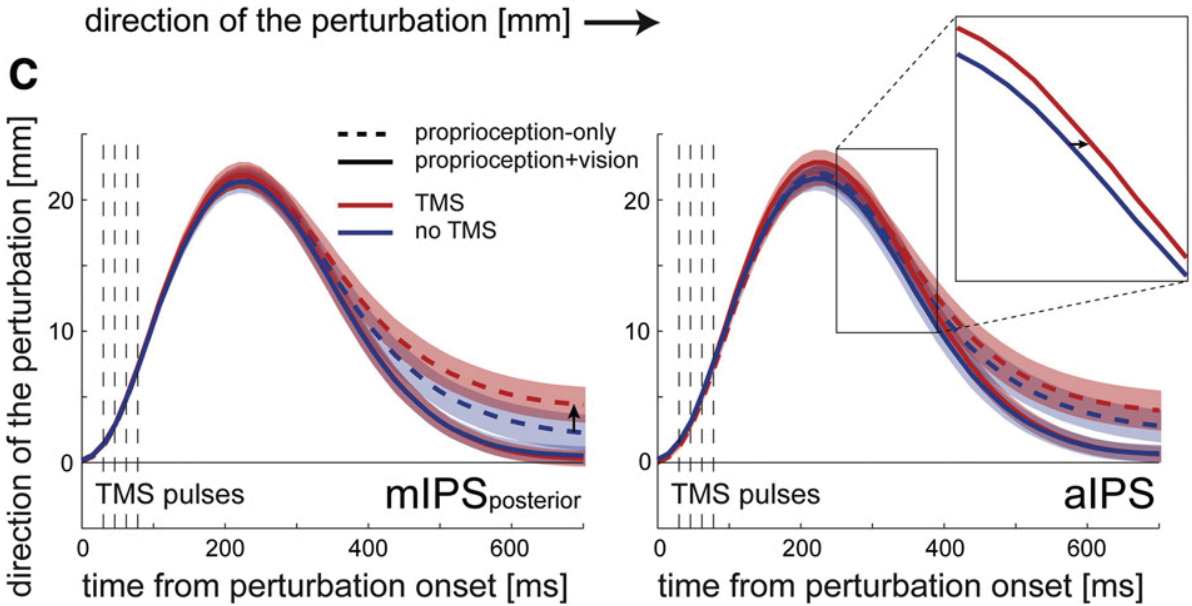

Fig. 3. Group trajectories illustrating the TMS effects for stimulation sites mIPS posterior $_{\text {and }}$ IIPS. A) Proprioception-only force perturbed trajectories normalized over path for TMS stimula-

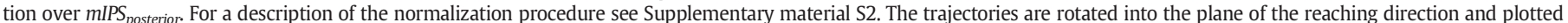
relative to the force perturbation (the force perturbation is directed to the positive x-direction). The left panel illustrates the whole reaching trajectory, while the right panel is a zoomed version to illustrate the TMS effect. Error bars denote standard error of the mean across participants at 10 equidistant sections of the trajectories. B) Proprioception + vision force perturbed trajectories normalized over time for TMS stimulation over aIPS. Conventions analogue to panel A. C) Trajectories over time averaged across all participants in the direction

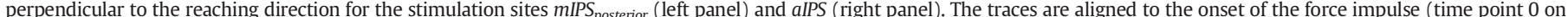
the $\mathrm{x}$-axis). The direction of the force impulse denotes positive values on the y-axis. Arrows illustrate the respective effects found in the group statistics (see also inset). The shaded areas denote standard error of the mean across participants.

668 ms (ReachTime; see Table 2 for details and statistical comparisons). Furthermore, it increased the endpoint variability $(\operatorname{Var} X)$ in direction of the force from 8.3 to $9.5 \mathrm{~mm}$, but decreased the variability in the direction of the reaching movement (VarY) from 10.2 to $9.2 \mathrm{~mm}$ (force perturbation effect in Table 2). Having visual feedback about the hand position available (sensory feedback effect in Table 2 for both Force- perturbed and Unperturbed) decreased the overshoot in the direction of the reaching movement (ErrorY), and decreased the variability both in direction of the force perturbation $(\operatorname{VarX})$ and in direction of the reaching movement (VarY). Applying TMS, however, yielded no general effect across TMS stimulation sites on any of those measurements (TMS effect in Table 2 for both Force-perturbed and Unperturbed). 
TMS over the posterior part of the mIPS (mIPS posterior $_{\text {) }}$ reduced the extent of corrective movements based on proprioception only

The main question addressed in the current study was whether any PPC area is crucial for processing proprioceptive information for online movement control. Comparing trials with vs. without TMS for proprioception-only reaching revealed that administering TMS over

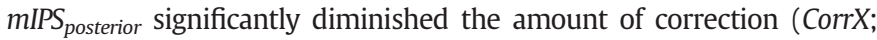
$t_{8}=3.69 ; p=.003$; permutation test threshold $=.007$; Figs. 3A\&C, $4 \mathrm{~A}$ white boxes). No other stimulation sites showed significant TMS effects (Fig. 4A white boxes and Supplementary material Table S2). Moreover, the effect from administering TMS over IIPS $_{\text {posterior }}$ was larger than the averaged effect from all other sites $\left(t_{8}=2.14 ; p<.05\right)$. Comparing the TMS effect for IIPS $_{\text {posterior }}$ with the effects for its adjacent stimulation sites mIPS $_{\text {middle }}\left(t_{8}=1.74 ; p=.06 ;\right.$ distance $8.1 \pm$ $0.05 \mathrm{~mm})$, alPS $_{\text {posterior }}\left(t_{8}=1.57 ; p=.08\right.$; distance $\left.14.9 \pm 0.10 \mathrm{~mm}\right)$, and $\mathrm{mIPS}_{\text {anterior }}\left(t_{8}=1.70 ; p=.06\right.$; distance $14.4 \pm 0.08 \mathrm{~mm}$ ) suggest that it its rather likely that the effect is specific for this cortical area. Moreover, the effect over mIPS posterior is functionally specific, as the TMS effect was significantly higher for proprioception-only than proprioception + vision reaching $\left(t_{8}=4.36 ; p=.001\right)$.

Administering TMS over any stimulation site did not change any other reaching parameter, for neither force-perturbed nor unperturbed reaches (CorrX, ErrorX, ErrorY, VarX, VarY, ReachTime, DecTime, AccTime;
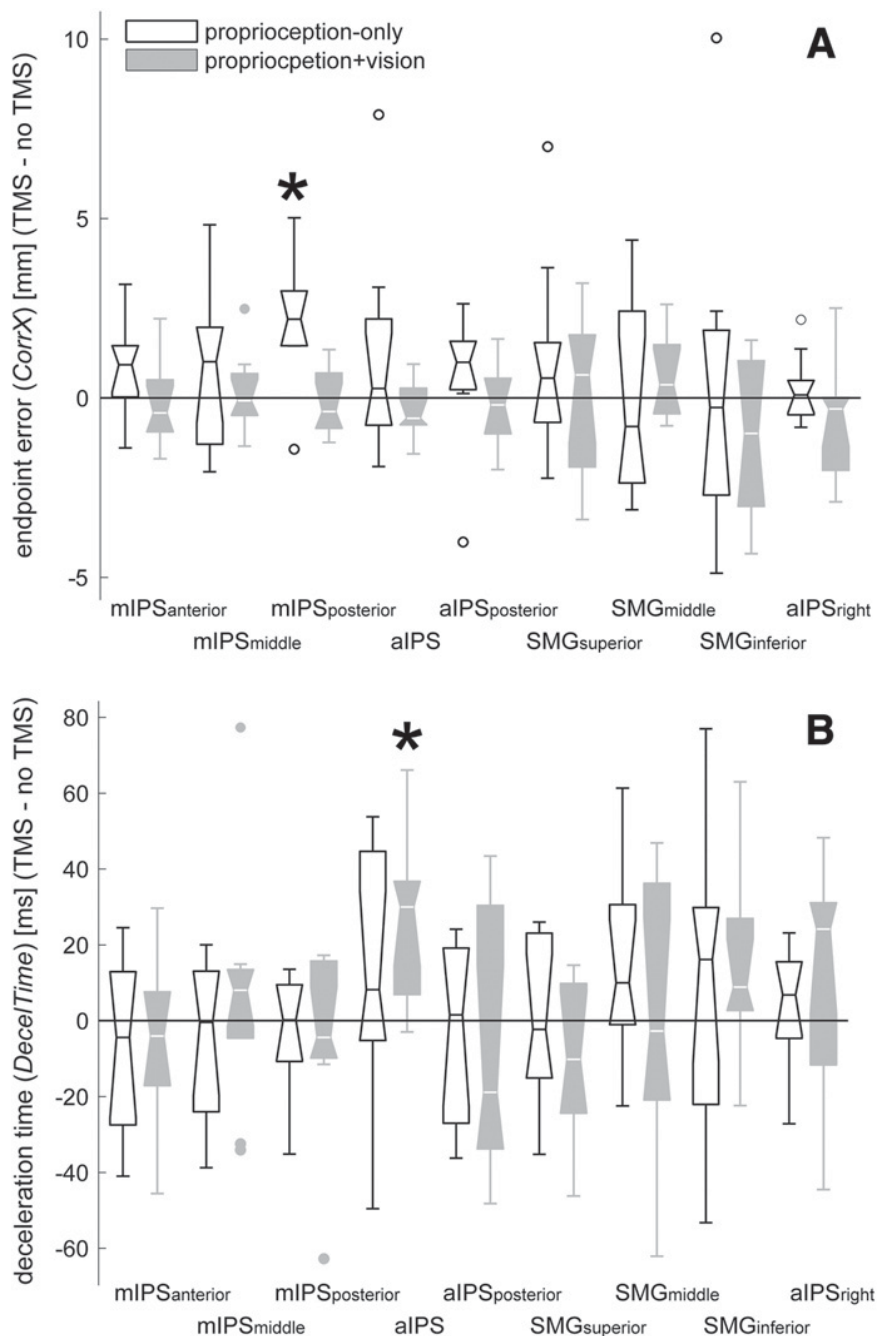

Fig. 4. Differences of end accuracy (CorrX, A) and deceleration time (DecelTime, B) for trials with TMS-trials without TMS. The boxes range from the 25th to the 75th percentile of the data, maximal whisker length is $1.5 \times$ box length (corresponds to approx. $99.3 \%$ if data is normal distributed). Outliers are indicated by circles. ${ }^{*} p<.05$, corrected. see Supplementary material Table S2 for details). Importantly, the acceleration period, the time in which the TMS pulses were administered, was also independent of TMS administration. Thus, TMS over any stimulation site did not alter the temporal movement dynamics during administration.

In summary, administering TMS over IIPS $_{\text {posterior }}$ specifically diminished the corrective response to the force perturbation when proprioception was the only sensory feedback available for detecting and correcting the perturbation. This became apparent through reduced endpoint correction in the direction of the perturbation (CorrX) exclusively when we administered TMS over IIPS $_{\text {posterior. }}$. Moreover, the results suggest that $m I P S_{\text {posterior }}$ plays a functional specific role in proprioception-only compared to proprioception + vision reaching.

TMS over the aIPS prolonged the deceleration period for corrective movements based on proprioception and vision

In contrast, TMS did not alter the endpoint accuracy when administered over any stimulation site for proprioception + vision reaching (see Fig. 4A grey boxes, Supplementary material Table S2 for details). We expected a good reaching accuracy for this condition (Spijkers and Lochner, 1994), and endpoint accuracy has already shown to be quite robust to TMS interference over the PPC for reaching with visual feedback about the hand position present (Reichenbach et al., 2011). However, TMS administered over aIPS significantly increased overall movement time (ReachTime; $t_{8}=3.33 ; p=.005$, permutation test threshold $=.006$ ), while there was no consistent effect of TMS on movements time for any other stimulation site. Specifically, the acceleration period, the time in which the TMS pulses were administered, remained unaltered for all stimulation sites (see Supplementary material Table S2 for details). The deceleration phase of the reaching movement, however, was significantly increased by TMS over aIPS (DecTime; $t_{8}=3.78 ; p=.003$, permutation test threshold $=.006$; Figs. $3 \mathrm{~B} \& \mathrm{C}, 4 \mathrm{~B}$ grey boxes). Furthermore, the increase in deceleration time for TMS over aIPS was significantly stronger than the average time effect for all other sites $\left(t_{8}=3.06 ; p<.01\right)$. Comparing the TMS effect for aIPS with the effects for its adjacent stimulation sites aIPS $_{\text {posterior }}\left(t_{8}=1.97 ; p<.05\right.$; distance $\left.7.4 \pm 0.03 \mathrm{~mm}\right), S M G_{\text {superior }}$ $\left(t_{8}=3.16 ; p<.01 ;\right.$ distance $\left.8.9 \pm 0.09 \mathrm{~mm}\right)$, and mIPS $_{\text {anterior }}$ $\left(t_{8}=3.38 ; p<.01\right.$; distance $\left.10.1 \pm 0.08 \mathrm{~mm}\right)$ clearly shows that the effect is specific for this cortical area. Yet, there is no functional specificity for the anterior IPS, as the effect for proprioception + vision reaching was not significantly higher than the effect for proprioception-only reaching $\left(t_{8}=0.95 ; p=.18\right)$. There was no effect of TMS over any other stimulation site or for any other measurement (CorrX, ErrorX, ErrorY, VarX, VarY, AccTime) for force-perturbed or unperturbed reaching based on proprioception + vision (see Supplementary material Table S2 for details).

In summary, administering TMS over aIPS specifically prolonged the deceleration phase (DecTime) of the movements perturbed with a force impulse when proprioception and vision could be used for detecting and correcting the perturbation. Moreover, our results suggest a good spatial specificity for this cortical area. In contrast, the functional specificity for the type of sensory feedback provided seems to be less pronounced.

\section{Discussion}

Our results show that TMS affects the correction for a force impulse administered to the reaching hand differentially at distinct sites of the left PPC, dependent on the availability of visual feedback about the hand position. TMS over the posterior part of the medial IPS ( IIPS $_{\text {posterior }}$ ) significantly disturbed endpoint accuracy in proprioception-only reaching. Specifically, when only proprioceptive information was available to detect and correct for the reaching error, administering TMS resulted in incomplete correction for the force perturbation. In contrast, 
TMS over the anterior IPS (aIPS) significantly prolonged the deceleration phase of the force-perturbed reaches in proprioception + vision reaching, where proprioceptive and visual information was available for estimating the current perturbed hand position. Importantly, the effects of TMS were only observed at distinct sites, and adjacent sites were not affected. Taken together, dissociable sub-regions of the left PPC play different roles in the processing of proprioceptive vs. multisensory information for online control of reaching.

The posterior part of the medial IPS is crucial for processing proprioceptive information about the reaching hand during an ongoing movement

TMS over the posterior part of the mIPS ( IIPS $_{\text {posterior }}$ ) markedly affected the ability to fully correct for the force impulse when proprioception was the only sensory information available for estimating the current hand position. The isolated effect on the spatial component of the trajectory in combination with its specificity for the proprioception-only condition strongly suggests that the TMS pulses over the posterior mIPS temporarily disturbed updating of the proprioceptive representation of the hand position. Consequentially, as re-calibration of proprioception with the spatial more precise visual representation was not possible, the internal representation of the actual hand position became increasingly imprecise when the hand deviated from the planned and executed trajectory due to the force impulse, leading to the observed endpoint error (Miall et al., 2007). Alternatively, it is possible that this area is important for processing a modality unspecific localization of the hand representation. In this case, TMS would not affect proprioception + vision reaching because the representation formed by the combination of both sensory modalities was more robust, or because visual information arriving after the stimulation was able to compensate for the TMS perturbation. If TMS had disturbed any other component of the sensorimotor processes, then the effect would not be specific for proprioception-only reaching, or the disruption would have rather caused a prolongation of the reach time for compensating the brief disruption in cortical processing. In any case, the posterior mIPS is critical for processing the hand position in the absence of visual feedback about the moving hand during the reach. This finding complements the studies based on which we included mIPS posterior into the current study. Della-Maggiore et al. (2004) have shown that TMS applied over this area also interferes with the learning of novel dynamics induced by a force field, and Chib et al. (2009) have demonstrated that this area is important for tracking the movement of a manipulandum in the absence of visual feedback. In both studies, processing of proprioceptive information was the key for successful task execution as in our proprioception-only condition. Together, these studies demonstrate that the human posterior mIPS is a key structure for processing proprioceptive hand/arm information for goal-directed movement control.

The TMS pulses were applied in a period from 30 to $90 \mathrm{~ms}$ after onset of the force perturbation. Since the onset of responses to such perturbations can be observed in less than $100 \mathrm{~ms}$ in EMG (Reichenbach et al., 2009), our stimulation period covered the whole time in which the parietal cortex could process information about the perturbation leading to an adjustment of the motor command. We cannot pinpoint though, at which time point exactly the posterior mIPS was crucial for information processing. TMS studies in primary visual (Amassian et al., 1989; Thielscher et al., 2010) and motor (Di Lazzaro et al., 1999; Ferbert et al., 1992) areas, and TMS connectivity studies between higher order and primary motor cortices (Koch et al., 2007; Mochizuki et al., 2004) succeeded in narrowing down chronometry of neural processing to time windows of few milliseconds. Those effects, however, are much more robust than TMS effects due to virtual lesions of higher cortical areas such as the posterior parietal cortex. Thus, we decided to sacrifice some temporal resolution for stability in effects. Moreover, we cannot exclude that some of the other areas might become more important than the posterior mIPS in later phases of the reach towards approaching the target. Investigations on movement preparation have revealed the temporal interplay between motor-related cortical areas before the movement starts (Davare et al., 2006; Koch et al., 2008, 2010). Along this line, the temporal involvement of the sensorimotor areas and their interaction throughout the evolving movement is an interesting topic for future studies.

While most fMRI studies agree that the medial IPS is involved in proprioceptive processing for motor control, the precise localization and nature of the processes is largely inconsistent. Studies directly concerned with processing of proprioceptive information during a movement report BOLD activation around our mIPS $_{\text {posterior, }}$ which extends more medially into the precuneus (Bernier and Grafton, 2010; Filimon et al., 2009; Pellijeff et al., 2006). However, this region has also been linked to other sensorimotor control processes (Blangero et al., 2009; Diedrichsen et al., 2005). All these studies utilized different experimental paradigms and comparisons have to be made with caution. Moreover, since the temporal resolution of fMRI is sub-optimal for studying fast online control processes, fMRI studies usually report BOLD activation resulting from a temporal mixture of sensorimotor processes. Other studies administering TMS over the mIPS to study reaching related processes have applied TMS during the planning phase, before the actual movement started (Busan et al., 2009, 2012; Davare et al., 2012; Vesia and Crawford, 2012; Vesia et al., 2006, 2008, 2010). The only exception is a study by Striemer et al. (2011). There, the authors contrasted a) the contribution of two sites (subsumed as superior parietal lobe (SPL), corresponding roughly to our anterior and posterior mIPS sites) to the planning $v s$. the execution phase of a proprioception-only reaching movement, and b) the contribution of the inferior parietal lobe vs. the SPL for reach planning and execution. Their finding that TMS over SPL only affects the planning but not the execution phase seems to contradict our result. However, since they used unperturbed reaching movements to a target extinguished at movement onset, participants probably relied on their initially formed motor plan for movement execution. These findings and another study investigating planning of a memory-guided proprioception-only reach by administering TMS over the mIPS (Vesia et al., 2010) suggest in combination with our result that proprioceptive information for planning and online control of reaching movements might be processed in the same or close by parietal areas, which is commonly referred to as medial IPS. Interestingly, the authors of the latter study suggest in a recent review (Vesia and Crawford, 2012) that the mIPS might be specific for the visual calculation of the reach vector, while the angular gyrus (AG) might be specific for the somatosensory reach vector. This suggestion also seems to contradict our result. However, it is based on an fMRI study investigating reaching after mirrorreversal adaptation (Fernandez-Ruiz et al., 2007), and a similar TMS study on the AG (Vesia et al., 2006). Both studies only investigated the planning phase of the reach, and the TMS study did not include the mIPS. While our data can neither shed light on the neural correlates of reach planning nor on the contribution of the $A G$ to this process, our result clearly shows the contribution of the posterior mIPS during online control of reaching. We demonstrated for the first time unambiguously the disruption of proprioceptive processing for the online control of reaching movements when administering TMS over the posterior part of the MIPS. However, we cannot exclude that the area processing this information extends more medially and/or posteriorly as IISS $_{\text {posterior }}$ was the most posterior site tested. Taken together, all these studies and our findings demonstrate that the posterior part of the mIPS, most probably extending medially to the precuneus according to the fMRI studies cited above, is important for various processes involving proprioception in motor control, including online control processes.

\section{Relevance of the observed TMS effect}

The reported spatial TMS effect ( $\sim 10 \%$ decrease in final correction) is smaller than e.g. the effect additional visual feedback has on the extent of the reaches or their endpoint variability. However, in the context of other parietal TMS studies in the field of motor control, it actually 
represents a substantial behavioral impairment. Studies demonstrating the necessity of the anterior IPS in grasping (Rice et al., 2006, 2007; Tunik et al., 2005, 2007) consistently report only changes in the temporal dynamics during the movement, but no spatial effects due to TMS. The studies of Chib et al. (2009) and Reichenbach et al. (2011) report spatial effects in the order of the effects reported here. The effects of Della-Maggiore et al. (2004) seem to accumulate up to 30\%. However, this constitutes an adaptation effect accumulated over the whole time course of the experiment, which started to manifest only after half of the session. We therefore consider the consistent under-correction of $\sim 10 \%$ due to TMS a severe impairment in the online control of movement.

The anterior IPS is involved in more general sensorimotor processes during voluntary movements

Administering TMS over the junction between the left postcentral and intraparietal sulcus (aIPS) significantly prolonged reaching time, in particular the deceleration phase, for force-perturbed proprioception + vision reaching. In this condition, the sensorimotor system could use proprioceptive and visual information to update the current hand position. TMS over the right aIPS or any other stimulation site on the left PPC yielded no effect. Providing visual feedback about the hand position yields rather good reaching accuracy (Spijkers and Spellerberg, 1995; Spijkers and Lochner, 1994). Given the transient effects of event-related TMS, the endpoint accuracy was not compromised by TMS as expected from a previous TMS reaching study (Reichenbach et al., 2011). Even though the extension of the reaching time was specific for force-perturbed trials, it was not functionally specific for reaching with visual information about the hand position present. Thus, we propose that TMS temporarily disturbed the integration of newly arriving sensory information (about the hand position) into the reaching plan, and catching up with the processing occupied time that was added to the reach time towards the end of the movement. Assuming that in this case vision was the main source of information for the corrective movement, this idea is in line with a previous study where administering TMS over aIPS delayed the onset of corrective movements to visual perturbations (Reichenbach et al., 2011). Along the same line, corrections in grasping movements to changes in target size or orientation can be disturbed by administering TMS over the aIPS (Rice et al., 2006, 2007; Tunik et al., 2005, 2007). Furthermore, Diedrichsen et al. (2005) have shown activation around the aIPS for execution errors during proprioception + vision reaching using a similar paradigm as in our study.

Given the prominent role of aIPS in grasping (Culham et al., 2003; Rice et al., 2006; Tunik et al., 2005), it is possible that the nature of our task, i.e. reaching movements while grasping a robotic manipulandum, had influenced our results. Furthermore, it has been shown that the spatiotemporal characteristics of free reaching movements vs. movements with a manipulandum differ (Desmurget et al., 1997). However, utilizing robotic manipulanda for recording and manipulating movements is quite common in reaching research (cf. references in Howard et al. (2009)), and especially experimental manipulations such as the force perturbation are not possible with free reaching. Since we only compared movements executed with a manipulandum, we do not believe that the spatial and temporal TMS effects observed stemmed from our particular experimental setup. Moreover, since the handle of the robot was grasped throughout the whole movement across all experimental conditions and no adjustment of the grasp was required at any time, it is unlikely that the TMS effects specific to the proprioception + vision condition arose from the circumstance that we tested reaching movements with a manipulandum.

Taken together, our finding adds to the notion of the left aIPS playing a key role in the processing of sensory information for online motor control beyond grasping (Tunik et al., 2007), the function mostly associated with the aIPS. Yet, the exact nature of the sensorimotor processing and a probable parceling of the area have to be identified in further studies.
For instance, the internal estimates about the target and hand position could be calculated here (Shadmehr and Krakauer, 2008), and the area could be subdivided into visual and proprioceptive, or target and hand sub-compartments.

\section{TMS grid measure increases the spatial specificity of the results}

Our results strongly suggest the existence of two functionally distinct, but neighboring parietal regions (anterior IPS and posterior part of the medial IPS): TMS stimulation over sites located in between these two areas yielded no effect on any of the reaching movements. Preferentially, we would have based the localization of the TMS stimulation sites on individual fMRI activations. However, due to the technical challenges with force perturbations in MRI, we opted for a standard approach that assesses group statistics based on brain areas transformed to the same MNI coordinates. In contrast to the majority of other TMS studies, however, we measured the behavioral effects over a densely spaced grid of TMS coil positions, which added some spatial specificity to our results. We did not include more medial-posterior areas into the grid as they have mainly been associated with processing information about the target of reach (Bernier and Grafton, 2010; Diedrichsen et al., 2005; Gallivan et al., 2009; Vesia et al., 2010), which was out of the scope of this study. A posteriori, this turned out to be a limitation of the current study since we found the main effect on the most posterior site tested. Thus, we can confine IIPS $_{\text {posterior }}$ only to more anterior areas but not to more posterior ones. Another general limitation of TMS, its restriction to localizing the anatomical areas of interest only with 2D focus since stronger electrical fields are always induced in more superficial areas, cannot be overcome with this approach. However, measuring a grid provides additional information to control for this problem. Demonstrating that optimal stimulation of adjacent, putatively more superficial sites does not result in behavioral impairment helps to rule out that the TMS caused a disruption of these areas rather than the targeted area. For interpreting the anatomical localization of TMS effects, it has to be kept in mind though, that superficial areas experience stronger electric fields than areas deep in sulci if both have the same 2D distance from the TMS stimulation site. For our stimulation sites, this means that stimulating mIPS $_{\text {posterior }}$ probably induced the strongest electrical field at the crown of the dorsal bank of the MIPS, and stimulating aIPS likely induced the strongest field at the crown of the ventral bank of the aIPS in the majority of our participants. It remains to be tested whether stimulating directly at these crowns also resulted in stronger physiological effects: The orientation of the neural structures relative to the induced field also differs between the different positions, which is a further factor which has to be taken into account (Opitz et al., 2011).

\section{Control for unspecific TMS effects}

Using individually adjusted stimulation intensities, we carefully controlled that TMS unlikely caused direct motor impairments that would have biased our results. This was confirmed by the specificity of the effects: General motor impairment or any other unspecific effect would have influenced all types of reaching to a similar extent, independent of the sensory feedback provided. In addition, the absence of TMS effects when stimulating adjacent sites with equal or closer proximity to the primary motor cortex render the possibility of any unspecific TMS effect very unlikely. The analysis of the acceleration-deceleration profiles served as additional control. The acceleration phase, which included the time period during which the TMS stimuli were delivered, was not altered by TMS stimulation over any site, not even on the site where the overall reaching time was prolonged (see Supplementary material Table S2 for details). Direct TMS effects on the motor cortex (e.g. muscle twitches and silent periods) have short latencies so that, in our case, general motor impairments by TMS should preferably affect the initial acceleration period (Desmurget et al., 1999). In general, the close proximity of adjacent stimulation sites and the specificity 
of TMS effects render the possibility of any other side effect, e.g. disruption of eye movements (also tested for explicitly in a previous study (Reichenbach et al., 2011)), very unlikely. To summarize, the observed TMS effects above mIPS posterior $_{\text {and }}$ aIPS demonstrate a specific disturbance of sensorimotor processing for the online corrections of the reaching movements, and cannot stem from unspecific TMS effects.

\section{Conclusions}

We demonstrated a causal contribution of two distinct sites in different processes for the control of reaching movements to a visual target using a grid of TMS coil positions over the anterior to middle part of the left PPC. The posterior part of the medial IPS is a key region for processing proprioceptive-only information during online motor control. In contrast, the anterior IPS seems to be more generally involved in the processing of sensory information for the control of movements.

\section{Acknowledgments}

This research was supported by a PhD stipend from the Max Planck Society and by the WCU (World Class University) program through the National Research Foundation of Korea funded by the Ministry of Education, Science and Technology (R31-2008-000-10008-0). The authors would like to thank Sonja Cornelsen for help in data acquisition, and Marc Himmelbach for helpful comments on a previous version of the manuscript.

\section{Appendix A. Supplementary material}

\section{Supplementary data to this article can be found online}

\section{References}

Amassian, V.E., Cracco, R.Q., Maccabee, P.J., Cracco, J.B., Rudell, A., Eberle, L., 1989. Suppression of visual perception by magnetic coil stimulation of human occipital cortex. Electroencephalogr. Clin. Neurophysiol. 74, 458-462.

Bernier, P.M., Grafton, S.T., 2010. Human posterior parietal cortex flexibly determines reference frames for reaching based on sensory context. Neuron 68, 776-788.

Bernier, P.M., Burle, B., Hasbroucq, T., Blouin, J., 2009. Spatio-temporal dynamics of reachrelated neural activity for visual and somatosensory targets. Neuroimage 47, 1767-1777.

Blangero, A., Menz, M.M., McNamara, A., Binkofski, F., 2009. Parietal modules for reaching. Neuropsychologia 47, 1500-1507.

Busan, P., Barbera, C., Semenic, M., Monti, F., Pizzolato, G., Pelamatti, G., Battaglini, P.P. 2009. Effect of transcranial magnetic stimulation (TMS) on parietal and premotor cortex during planning of reaching movements. PLoS One 4, e4621.

Busan, P., Zanon, M., Vinciati, F., Monti, F., Pizzolato, G., Battaglini, P.P., 2012. Transcranial magnetic stimulation and preparation of visually-guided reaching movements. Front. Neuroeng. 5, 18 .

Buss, M., Schmidt, G., 1999. Control problems in multimodal telepresence systems. Advances in Control, Highlights of ECC '99. Springer-Verlag, London, pp. 65-101.

Chib, V.S., Krutky, M.A., Lynch, K.M., Mussa-Ivaldi, F.A., 2009. The separate neural control of hand movements and contact forces. J. Neurosci. 29, 3939-3947.

Culham, J.C., Valyear, K.F., 2006. Human parietal cortex in action. Curr. Opin. Neurobiol. 16, 205-212.

Culham, J.C., Danckert, S.L., DeSouza, J.F., Gati, J.S., Menon, R.S., Goodale, M.A., 2003. Visually guided grasping produces fMRI activation in dorsal but not ventral stream brain areas. Exp. Brain Res. 153, 180-189.

Culham, J.C., Cavina-Pratesi, C. Singhal, A., 2006. The role of parietal cortex in visuomotor control: what have we learned from neuroimaging? Neuropsychologia 44, 2668-2684.

Davare, M., Andres, M., Cosnard, G., Thonnard, J.L., Olivier, E., 2006. Dissociating the role of ventral and dorsal premotor cortex in precision grasping. J. Neurosci. 26, 2260-2268.

Davare, M., Zenon, A Pourtois, G. Desmurget, M., Olivier, E, 2012. Role of the medial part of the intraparietal sulcus in implementing movement direction. Cereb. Cortex 22 , $1382-1394$.

Della-Maggiore, V., Malfait, N., Ostry, D.J., Paus, T., 2004. Stimulation of the posterior parietal cortex interferes with arm trajectory adjustments during the learning of new dynamics. J. Neurosci. 24, 9971-9976.

Desmurget, M., Grafton, S., 2000. Forward modeling allows feedback control for fast reaching movements. Trends Cogn. Sci. 4, 423-431.

Desmurget, M., Jordan, M., Prablanc, C., Jeannerod, M., 1997. Constrained and unconstrained movements involve different control strategies. J. Neurophysiol. 77, $1644-1650$.
Desmurget, M., Epstein, C.M., Turner, R.S., Prablanc, C., Alexander, G.E., Grafton, S.T., 1999. Role of the posterior parietal cortex in updating reaching movements to a visual target. Nat. Neurosci. 2, 563-567.

Di Lazzaro, V., Oliviero, A., Profice, P., Insola, A., Mazzone, P., Tonali, P., Rothwell, J.C., 1999. Direct demonstration of interhemispheric inhibition of the human motor cortex produced by transcranial magnetic stimulation. Exp. Brain Res. 124, 520-524.

Diedrichsen, J., Hashambhoy, Y., Rane, T., Shadmehr, R., 2005. Neural correlates of reach errors. J. Neurosci. 25, 9919-9931.

Ferbert, A., Priori, A., Rothwell, J.C., Day, B.L., Colebatch, J.G., Marsden, C.D., 1992 Interhemispheric inhibition of the human motor cortex. J. Physiol. 453, 525-546.

Fernandez-Ruiz, J., Goltz, H.C., DeSouza, J.F., Vilis, T., Crawford, J.D., 2007. Human parietal "reach region" primarily encodes intrinsic visual direction, not extrinsic movement direction, in a visual motor dissociation task. Cereb. Cortex 17, 2283-2292.

Filimon, F., Nelson, J.D., Huang, R.S., Sereno, M.I., 2009. Multiple parietal reach regions in humans: cortical representations for visual and proprioceptive feedback during online reaching. J. Neurosci. 29, 2961-2971.

Gallivan, J.P., Cavina-Pratesi, C., Culham, J.C., 2009. Is that within reach? fMRI reveals that the human superior parieto-occipital cortex encodes objects reachable by the hand. J. Neurosci. 29, 4381-4391.

Glover, S., 2002. Visual illusions affect planning but not control. Trends Cogn. Sci. 6, 288-292.

Goodale, M.A., Pelisson, D., Prablanc, C., 1986. Large adjustments in visually guided reaching do not depend on vision of the hand or perception of target displacement. Nature 320, 748-750.

Heed, T., Beurze, S.M., Toni, I., Roder, B., Medendorp, W.P., 2011. Functional rather than effector-specific organization of human posterior parietal cortex. J. Neurosci. 31, 3066-3076.

Howard, I.S., Ingram, J.N., Wolpert, D.M., 2009. A modular planar robotic manipulandum with end-point torque control. J. Neurosci. Methods 181, 199-211.

Iacoboni, M., 2006. Visuo-motor integration and control in the human posterior parietal cortex: evidence from TMS and fMRI. Neuropsychologia 44, 2691-2699.

Kammer, T., Vorwerg, M., Herrnberger, B., 2007. Anisotropy in the visual cortex investigated by neuronavigated transcranial magnetic stimulation. Neuroimage 36, 313-321.

Koch, G., Fernandez Del Olmo, M., Cheeran, B., Ruge, D., Schippling, S., Caltagirone, C., Rothwell, J.C., 2007. Focal stimulation of the posterior parietal cortex increases the excitability of the ipsilateral motor cortex. J. Neurosci. 27, 6815-6822.

Koch, G., Fernandez Del Olmo, M., Cheeran, B., Schippling, S., Caltagirone, C., Driver, J., Rothwell, J.C., 2008. Functional interplay between posterior parietal and ipsilateral motor cortex revealed by twin-coil transcranial magnetic stimulation during reach planning toward contralateral space. J. Neurosci. 28, 5944-5953.

Koch, G., Cercignani, M., Pecchioli, C., Versace, V., Oliveri, M., Caltagirone, C., Rothwell, J., Bozzali, M., 2010. In vivo definition of parieto-motor connections involved in planning of grasping movements. Neuroimage 51, 300-312.

Krakauer, J.W., Ghilardi, M.F., Ghez, C., 1999. Independent learning of internal models for kinematic and dynamic control of reaching. Nat. Neurosci. 2, 1026-1031.

Mazziotta, J., Toga, A., Evans, A., Fox, P., Lancaster, J., Zilles, K., Woods, R., Paus, T., Simpson, G., Pike, B., Holmes, C., Collins, L., Thompson, P., MacDonald, D., Iacoboni, M., Schormann, T., Amunts, K., Palomero-Gallagher, N., Geyer, S., Parsons, L., Narr, K., Kabani, N., Le Goualher, G., Boomsma, D., Cannon, T., Kawashima, R., Mazoyer, B., 2001. A probabilistic atlas and reference system for the human brain: International Consortium for Brain Mapping (ICBM). Philos. Trans. R. Soc. Lond. B Biol. Sci. 356, $1293-1322$

Miall, R.C., Christensen, L.O., Cain, O., Stanley, J., 2007. Disruption of state estimation in the human lateral cerebellum. PLoS Biol. 5, e316.

Mochizuki, H., Huang, Y.Z., Rothwell, J.C., 2004. Interhemispheric interaction between human dorsal premotor and contralateral primary motor cortex. J. Physiol. Lond. 561, 331-338.

Oliver, R., Bjoertomt, O., Driver, J., Greenwood, R., Rothwell, J., 2009. Novel 'hunting' method using transcranial magnetic stimulation over parietal cortex disrupts visuospatial sensitivity in relation to motor thresholds. Neuropsychologia 47, 3152-3161.

Opitz, A., Windhoff, M., Heidemann, R.M., Turner, R., Thielscher, A., 2011. How the brain tissue shapes the electric field induced by transcranial magnetic stimulation. Neuroimage 58, 849-859.

Pellijeff, A., Bonilha, L., Morgan, P.S., McKenzie, K., Jackson, S.R., 2006. Parietal updating of limb posture: an event-related fMRI study. Neuropsychologia 44, 2685-2690.

Reichenbach, A., Thielscher, A., Peer, A., Bulthoff, H.H., Bresciani, J.P., 2009. Seeing the hand while reaching speeds up on-line responses to a sudden change in target position. J. Physiol. 587, 4605-4616.

Reichenbach, A., Bresciani, J.P., Peer, A., Bulthoff, H.H., Thielscher, A., 2011. Contributions of the PPC to online control of visually guided reaching movements assessed with fMRI-guided TMS. Cereb. Cortex 21, 1602-1612.

Rice, N.J., Tunik, E., Grafton, S.T., 2006. The anterior intraparietal sulcus mediates grasp execution, independent of requirement to update: new insights from transcranial magnetic stimulation. J. Neurosci. 26, 8176-8182.

Rice, N.J., Tunik, E., Cross, E.S., Grafton, S.T., 2007. On-line grasp control is mediated by the contralateral hemisphere. Brain Res. 1175, 76-84.

Sabes, P.N., 2000. The planning and control of reaching movements. Curr. Opin. Neurobiol. 10, $740-746$.

Sarlegna, F., Blouin, J., Bresciani, J.P., Bourdin, C., Vercher, J.L., Gauthier, G.M., 2003. Target and hand position information in the online control of goal-directed arm movements. Exp. Brain Res. 151, 524-535.

Sarlegna, F., Blouin, J., Vercher, J.L., Bresciani, J.P., Bourdin, C., Gauthier, G.M., 2004. Online control of the direction of rapid reaching movements. Exp. Brain Res. 157, 468-471.

Shadmehr, R., Krakauer, J.W., 2008. A computational neuroanatomy for motor control. Exp. Brain Res. 185, 359-381. 
Shadmehr, R., Smith, M.A., Krakauer, J.W., 2010. Error correction, sensory prediction, and adaptation in motor control. Annu. Rev. Neurosci. 33, 89-108.

Smith, S.M., Jenkinson, M., Woolrich, M.W., Beckmann, C.F., Behrens, T.E., Johansen-Berg H., Bannister, P.R., De Luca, M., Drobnjak, I., Flitney, D.E., Niazy, R.K., Saunders, J. Vickers, J., Zhang, Y., De Stefano, N., Brady, J.M., Matthews, P.M., 2004. Advances in functional and structural MR image analysis and implementation as FSL. Neuroimage 23 (Suppl. 1), S208-S219.

Smith, S.M., Beckmann, C.F., Ramnani, N., Woolrich, M.W., Bannister, P.R., Jenkinson, M., Matthews, P.M., McGonigle, D.J., 2005. Variability in fMRI: a re-examination of inter-session differences. Hum. Brain Mapp. 24, 248-257.

Spijkers, W.A., Lochner, P., 1994. Partial visual feedback and spatial end-point accuracy of discrete aiming movements. J. Mot. Behav. 26, 283-295.

Spijkers, W., Spellerberg, S., 1995. On-line visual control of aiming movements? Acta Psychol. (Amst.) 90, 333-348.

Striemer, C.L., Chouinard, P.A., Goodale, M.A., 2011. Programs for action in superior parietal cortex: a triple-pulse TMS investigation. Neuropsychologia 49, 2391-2399.

Tagliabue, M., McIntyre, J., 2011. Necessity is the mother of invention: reconstructing missing sensory information in multiple, concurrent reference frames for eye-hand coordination. J. Neurosci. 31, 1397-1409.
Thielscher, A., Reichenbach, A., Ugurbil, K., Uludag, K., 2010. The cortical site of visual suppression by transcranial magnetic stimulation. Cereb. Cortex 20, 328-338.

Tunik, E., Frey, S.H., Grafton, S.T., 2005. Virtual lesions of the anterior intraparietal area disrupt goal-dependent on-line adjustments of grasp. Nat. Neurosci. 8, 505-511.

Tunik, E., Rice, N.J., Hamilton, A., Grafton, S.T., 2007. Beyond grasping: representation of action in human anterior intraparietal sulcus. Neuroimage 36 (Suppl. 2), T77-T86.

Vesia, M., Crawford, J.D., 2012. Specialization of reach function in human posterior parietal cortex. Exp. Brain Res. 221, 1-18.

Vesia, M., Monteon, J.A., Sergio, L.E., Crawford, J.D., 2006. Hemispheric asymmetry in memory-guided pointing during single-pulse transcranial magnetic stimulation of human parietal cortex. J. Neurophysiol. 96, 3016-3027.

Vesia, M., Yan, X., Henriques, D.Y., Sergio, L.E., Crawford, J.D., 2008. Transcranial magnetic stimulation over human dorsal-lateral posterior parietal cortex disrupts integration of hand position signals into the reach plan. J. Neurophysiol. 100, 2005-2014.

Vesia, M., Prime, S.L, Yan, X Sergio, LE, Crawford, J.D., 2010. Specificity of human parietal saccade and reach regions during transcranial magnetic stimulation. J. Neurosci. 30 13053-13065.

Wolpert, D.M., Ghahramani, Z., Jordan, M.I., 1995. An internal model for sensorimotor integration. Science 269, 1880-1882. 